\title{
Development of a Kinetic Assay for Late Endosome Movement
}

\author{
Milan Esner 1,5, Felix Meyenhofer ${ }^{2,5}$, Michael Kuhn³, \\ Melissa Thomas ${ }^{4,5}$, Yannis Kalaidzidis ${ }^{5}$, and Marc Bickle ${ }^{5}$
}

\begin{abstract}
Automated imaging screens are performed mostly on fixed and stained samples to simplify the workflow and increase throughput. Some processes, such as the movement of cells and organelles or measuring membrane integrity and potential, can be measured only in living cells. Developing such assays to screen large compound or RNAi collections is challenging in many respects. Here, we develop a live-cell high-content assay for tracking endocytic organelles in medium throughput. We evaluate the added value of measuring kinetic parameters compared with measuring static parameters solely. We screened 2000 compounds in U-2 OS cells expressing Lampl-GFP to label late endosomes. All hits have phenotypes in both static and kinetic parameters. However, we show that the kinetic parameters enable better discrimination of the mechanisms of action. Most of the compounds cause a decrease of motility of endosomes, but we identify several compounds that increase endosomal motility. In summary, we show that kinetic data help to better discriminate phenotypes and thereby obtain more subtle phenotypic clustering.
\end{abstract}

\section{Keywords}

live cell, tracking, high-content imaging, Lampl, cardiac glycoside

\section{Introduction}

Live-cell imaging is a powerful analytical tool yielding information about the kinetics of biological processes or compound activity and allows for discrimination between primary and secondary phenotypes to correctly determine their mode of action. Nevertheless, most high-content screening assays are end-point assays as live-cell imaging is generally considered more challenging in high-throughput than fixed-cell imaging. Some examples of added difficulties of live-cell screening are maintaining sterile culture conditions, bleaching/phototoxicity issues during imaging, and devising schemes for adding compounds across many plates in a synchronous fashion to the acquisition to ensure equal incubation times across all wells. Therefore, only a few live-cell screens have been reported to date. ${ }^{1-4}$ Nevertheless, imaging screens are in some aspects easier to perform with live cells compared with fixed cells since neither fixing nor washing is required, thereby streamlining robotic workflows and reducing variability.

We took the challenge to bring an assay tracking subcellular objects to a medium-throughput assay. We developed an assay measuring the dynamics of late endosomes labeled with LAMP1-GFP in U-2 OS cells. LAMP1 is concentrated on late endosomes and lysosomes (Suppl. Fig. S1) ${ }^{5-7}$ This assay is amenable to screen several thousands of compounds and offers deeper insight into the mode of action of compounds compared with a fixed-cell assay. Here we present methods on how to optimize the settings for automated microscopy with live cells, add compounds in a nearly synchronous fashion to all wells, and analyze the resulting images to extract quantitative information about vesicle movement. We show that the kinetic parameters add critical dimensionality, allowing better phenotypic clustering than using static parameters only. Thanks to kinetic parameters, we identified a group of compounds from the family of cardiac glycosides, which increase the motion of endosomes.

\footnotetext{
'Department of Histology and Embryology, Faculty of Medicine, Masaryk University, Brno, Czech Republic

2University of Fribourg, Department of Medicine-Anatomy, Fribourg, Switzerland

${ }^{3}$ Biotechnology Center, TU Dresden, Dresden, Germany

${ }^{4}$ St Vincent's Centre for Applied Medical Research, Sydney, Australia

5Max-Planck Institute of Cell Biology and Genetics, Dresden, Germany
}

Supplementary material for this article is available

\section{Corresponding Author:}

Marc Bickle, Max-Planck Institute of Cell Biology and Genetics,

Pfotenhauerstrasse 108, Dresden, 01307, Germany.

Email: bickle@mpi-cbg.de 
All the other compounds have the opposite phenotype, leading to decreased movement of endosomes.

\section{Materials and Methods}

\section{Cell Culture}

U-2 OS cells were grown in RPMI 1640 Glutamax media with phenol red (cat. 61870; Invitrogen, Carlsbad, CA) supplemented with 10\% fetal calf serum (FCS) (cat. P282710; PAN, Aidenbach, Germany) and $50 \mu \mathrm{g} / \mathrm{mL}$ gentamicin (cat. 15750-037; Invitrogen). U-2 OS cells with Lamp1-GFP growth medium was supplemented with $200 \mu \mathrm{g} / \mathrm{ml}$ G418 (Cat. 10131-35, Invitrogen)

For experiments in microtiter plates, cells were first stained by adding Hoechst 33342 (cat. H21492; Invitrogen) at a final concentration of $2 \mu \mathrm{g} / \mathrm{mL}$ to a cell culture flask for $30 \mathrm{~min}$. After incubation, cells were washed twice with phosphate-buffered saline (PBS), dissociated with $0.05 \%$ trypsin (cat. 25300-054; Invitrogen) for $3 \mathrm{~min}$, counted with the CASY counter (Scharfe System, Reutlingen, Germany), and seeded at 4000 cells per well in $30 \mu \mathrm{L}$ RPMI 1640 without phenol red (cat. 32404; Invitrogen), supplemented with 2 mM L-glutamine (cat. 25030; Invitrogen), 25 mM HEPES (cat. 15630-049; Invitrogen), $50 \mu \mathrm{g} / \mathrm{mL}$ gentamicin, and $10 \%$ FCS into a 384-well imaging plate (cat. 781092; Greiner BioOne, Monroe, NC). Prior to acquisition, $40 \mu \mathrm{L}$ of mineral oil (cat. M8410; Sigma, St. Louis, MO) was added to each well to eliminate evaporation during acquisition.

\section{Chemical Library and Layout}

We screened the Spectrum Collection library from MicroSource Discovery (Gaylordsville, CT). This library contains approximately 2000 biologically active and structurally diverse compounds. Columns 2, 3, 4 and 23 contain negative and positive controls and untreated samples. Negative control was DMSO (cat. 276855; Sigma), and positive control was $5 \mu \mathrm{M}$ nocodazole (cat. M1404; Sigma) in DMSO. The library was distributed in seven 384-well plates and screened at $10 \mu \mathrm{M}$ in $0.1 \%$ DMSO final concentration.

\section{Image Acquisition}

Control movies to determine sampling parameters were acquired on an Olympus IX71 inverted wide-field microscope with an Olympus UApo/340 - 40×/0.9 objective (Olympus, Tokyo, Japan) and equipped with an Andor iXon EM DU 897 BV back-illuminated EMCCD camera (Andor, Belfast, Northern Ireland). This camera has $512 \times 512$ pixels, with a pixel size of $16 \mu \mathrm{m}$. With $40 \times$ lens and $2 \times$ optovar, the image pixel size is $0.2 \mu \mathrm{m}$.

All other images were acquired on an automated microscope (BD Biosciences Pathway 855; BD Biosciences, San Jose, CA) equipped with a live-cell chamber, using an
Olympus UApo/340 - $40 \times / 0.90$ objective with the filter sets shown in Supplementary Table S1.

The pixel size of the CCD camera was $6.45 \mu \mathrm{m}$. We acquired images with a binning of 2 , resulting in $0.323 \mu \mathrm{m} /$ pixel.

\section{Image Analysis}

The image analysis was performed with the freely available in-house developed software MotionTracking (http:// motiontracking.mpi-cbg.de/get/).

Image correction. We acquired reference images after each acquisition to correct images in a preprocessing step. The correction function is composed of a linear affine transformation (translation, rotation, projection) and a nonlinear spline fit for fine adjustment. ${ }^{8}$

The background is estimated with a simple mean filter with a kernel size approximately four times the size of the objects to be detected. The resulting image is then subtracted from the original.

In fluorescence microscopy, the predominant noise is the photon counting noise that follows a Poisson distribution and whose amplitude is proportional to the signal intensity. Linear models can be used to estimate noise amplitude as a function of the pixel intensity to weigh each pixel according to its information value. ${ }^{9}$

Image quantification. Segmentation of objects was achieved by fitting Lorenzian functions to find nuclei and endosomes, allowing determining intensity and shape parameters with high precision at a subpixel resolution. ${ }^{10}$ The cells were segmented with an active contour algorithm on the combined image of the endosome and nuclear channel. ${ }^{11}$ Static parameters were extracted from the segmented endosomes and nuclei.

For tracking, we used information about endosomal position, intensity, and shape to search locally for the best candidate for linking objects between frames. ${ }^{12}$ From the endosome tracks, we extracted kinetic parameters such as endosome speed, processivity, and displacement.

\section{Data Analysis}

Image analysis data were further processed with MATLAB from MathWorks (Natick, MA). Out-of-focus movies (low nuclei contrast and gradient intensity on the object edges) and movies containing either less than 3 or more than 35 cells were removed from analysis. Movies were considered outliers and removed if one of their measurements was more than 4 standard deviations (SD) away from the population mean within the same condition and the same plate. The parameters of the remaining movies were normalized by applying a platewise $z$ score normalization to DMSO. ${ }^{13,14}$ The median of the $z$ scores per well was then computed. In live-cell 
imaging, compounds are not removed before imaging and autofluorescent compounds can be problematic. Compounds yielding either nuclear Hoechst fluorescence more than 10 SD or GFP Lamp1 fluorescence more than 4 SD from DMSO were considered autofluorescent. Compounds were considered toxic if their volume-weighted Hoechst nuclear intensity was 3 SD above DMSO and the ratio of the cell to nuclei areas was 4 SD above DMSO. Autofluorescent and toxic compounds were removed from the hit list and not used for validation screen. For hierarchical clustering, $\mathrm{Z}$ score values were length normalized onto the unit hypersphere.

Principal component analysis and hierarchical clustering were performed in open-source programming language $\mathrm{R}$, version 2.14.0 and following versions (R Foundation for Statistical Computing, Vienna, Austria). Some graphs were produced using the ggplot2 library.

Parameters. Twenty-three of 96 measured Lamp1 parameters were chosen for analysis based on three criteria: (1) a Pearson correlation coefficient among parameters below 0.8 (absolute mean 0.225), (2) a Pearson correlation coefficient between runs above 0.8 , and (3) a heuristic that parameters representing all categories (intensity, morphology, location, kinetic) should be present. Furthermore, nuclei intensity morphology was measured. A point in an endosome track is called "processive" if it is followed by three consecutive points that fall between two rays forming an angle of 30 degrees, drawn from the first point. Subsequent points classified as "processive" form subsets of endosome tracks that are defined as processive movement events. A list with parameter names and their respective IDs is included in Table 1.

\section{Results}

\section{Establishing the Lamp I-GFP Cell Line}

To develop our endosome tracking assays, we established a stable cell line expressing a Lamp1-GFP construct under the control of the cytomegalovirus (CMV) promoter (see supplementary materials and Falcon-Perez et al. ${ }^{15}$ ).

\section{Determination of Image Acquisition Parameters}

To estimate our required sampling rate and to establish a minimal observation time, we first determined the speed of the Lamp1-GFP labeled endosomes. We acquired control movies at frame rates ranging from 2 to 30 frames per second (fps) for $60 \mathrm{~s}$ on a wide-field inverted microscope equipped with a fast back-illuminated EMCCD camera. We calculated the mode of the speed of endosomes for different frame rates of the control movies (Fig. 1A).

Another factor besides speed of objects influencing the necessary frame rate for tracking is the distance between objects. Densely distributed objects require faster frame rates compared with more sparse objects, as the distance traveled by an object between two frames can more readily exceed the object-to-object distance, potentially causing errors in object linking between frames. We therefore measured the nearest neighbor distances between objects (Fig. 1B) and estimated the maximum speed from a single movie.

We chose the mean of the nearest neighbor distance distribution divided by the maximum speed to obtain the frame rate (equation 1).

$$
\frac{\frac{\text { mean }\left\{d_{n n}\right\}}{2}}{v_{\max }}=\operatorname{mean}\left\{d_{f}(n)\right\} \approx \frac{0.9 \mu \mathrm{m}}{7 \mu \mathrm{m} / \mathrm{s}}=0.13 \mathrm{~s} .
$$

We determined that the maximal acquisition rate of the ORCA-ER CCD camera of our screening microscope at binning 2 was approximately $4.5 \mathrm{fps}$. Given the experimentally measured endosome speed and density, we determined that we were able to capture approximately $75 \%$ of all tracks but were not able to track the fastest events accurately.

To accurately estimate the kinetic parameters of objects, one needs to sufficiently sample them in time. We therefore aimed to establish the minimal number of images per movie necessary to obtain stable readouts. Several movies of $30 \mathrm{~s}$ length were acquired at the maximal frame rate of $4.5 \mathrm{fps}$. We calculated the mode of endosome speed for the entire sequences and shorter subsets to obtain different sampling intervals. The sampling interval yielding the minimal difference between the maximum deviation of individual measurements and the mean measurement for each movie was determined (Fig. 1C). These calculations resulted in a minimum movie length of $15 \mathrm{~s}$ with 68 images per movie. To have a safety margin, we decided to acquire 80 images per movie (approximately $19 \mathrm{~s}$ ).

A further important setting is the exposure time of each image, since it determines the signal-to-noise ratio. We created a set of artificial images designed to resemble actual cells with a known number of cell objects and artificial endosomes with variable intensities and variable signal-tonoise ratios. We then tested our image-processing software and set a minimal threshold detection accuracy of $95 \%$ to determine the lowest acceptable signal-to-noise ratio. A similar signal-to-noise ratio was attained in our images with exposure times of $150 \mathrm{~ms}$.

The minimal observation time, signal-to-noise ratio, and acquisition speed determine an acquisition parameter space, where the image analysis can be performed meaningfully. The observation time, signal-to-noise ratio, and acquisition speed are controlled on the microscope by setting the number of frames, exposure time, and the frame rate, respectively (see Fig. 1D). These constraints need to be respected when optimizing other assay parameters such as cell viability.

Last, a single image of the Hoechst-stained nuclei was acquired per field of view to estimate directionality of endosome movement, estimate compound toxicity, and focus 
Table I. Complete Names of All Measured Parameters and Their Corresponding Parameter ID.

\begin{tabular}{|c|c|}
\hline Parameter ID & Parameter Name \\
\hline I & Mean Speed (Channel I) ColorVesiculeSet Weighted=FALSE CalcType = Mean \\
\hline 2 & Mean of Max. Displacement (Channel I) ColorVesiculeSet Weighted=FALSE CalcType = Mean \\
\hline 3 & $\begin{array}{l}\text { Mean of Max. Displacement (Channel I) ColorVesiculeSet Weighted=TRUE WeightingFunc=GetVolume } \\
\text { CalcType = Mean }\end{array}$ \\
\hline 4 & $\begin{array}{l}\text { Mean Processive Movement (Channel I) ColorVesiculeSet Weighted=TRUE WeightingFunc=GetMeanIntensity } \\
\text { CalcType = Mean }\end{array}$ \\
\hline 5 & Mean of Processive to Total Time Ratio (Channel I) ColorVesiculeSet Weighted=FALSE CalcType = Mean \\
\hline 6 & $\begin{array}{l}\text { Mean of Processive to Total Time Ratio }(\text { Channel I) ColorVesiculeSet Weighted=TRUE } \\
\text { WeightingFunc=GetVolume CalcType = Mean }\end{array}$ \\
\hline 7 & Integ. Ves. Intens. (Channel I) ColorVesiculeSet Mask = TRUE \\
\hline 8 & MeanlntCHI \\
\hline 9 & MeanlntCHIVolWeig \\
\hline 10 & MeanlntegIntCHI \\
\hline 11 & MeanAreaCHI \\
\hline 12 & $\begin{array}{l}\text { Mean Anisotropy In Cells (Channel I) ColorVesiculeSet Weighted=FALSE WeighingFunc=GetVolume } \\
\text { ExcludeBorder=TRUE Normalize Size=TRUE CalcType = Mean }\end{array}$ \\
\hline 13 & $\begin{array}{l}\text { Dist. Distr. (Channel I) ColorVesiculeSet Min. Distance=0.5 Max. Distance=30 Weighted=FALSE } \\
\text { WeighingFunc }=\text { GetVolume Param I }\end{array}$ \\
\hline 14 & Integ. Ves. Intens. (Channel 2) ColorVesiculeSet Mask = TRUE \\
\hline 15 & MeanlntegIntCH2 \\
\hline 16 & MeanlntegIntCH2VolWeig \\
\hline 17 & MeanElongation $\mathrm{CH} 2$ \\
\hline 18 & $\begin{array}{l}\text { Per Cell Frame Intens. (Channel I) ColorVesiculeSet Intensity=Total NormalizeCellArea=TRUE Print To } \\
\text { File=FALSE CalcType = Mean }\end{array}$ \\
\hline 19 & Numb. Ves. per Cell (Channel I) ColorVesiculeSet Mask = TRUE Print To File=FALSE CalcType = Mean \\
\hline 20 & $\begin{array}{l}\text { Dist. to Nucl. Cell Based (Channel I) ColorVesiculeSet Weighted=FALSE WeighingFunc=GetVolume } \\
\text { Normalization=None CalcMethod=DistToNuc CalcType = Mean }\end{array}$ \\
\hline 21 & $\begin{array}{l}\text { Dist. to Nucl. Per Cell (Channel I) ColorVesiculeSet Weighted Vesicules=FALSE WeighingFuncVes=GetVolume } \\
\text { Weighted Cells=FALSE WeightingCellMethod=GetVolume Normalized=TRUE NormalizingFactor=NucleusSize } \\
\text { Print To File=FALSE CalcType = Mean }\end{array}$ \\
\hline 22 & $\begin{array}{l}\text { Dist. to Nucl. Per Cell (Channel I) ColorVesiculeSet Weighted Vesicules=FALSE WeighingFuncVes=GetVolume } \\
\text { Weighted Cells=FALSE WeightingCellMethod=GetVolume Normalized=TRUE NormalizingFactor=CellArea } \\
\text { Print To File=FALSE CalcType = Mean }\end{array}$ \\
\hline 23 & $\begin{array}{l}\text { Contact Ratio ColorVesiculeSet Weighted=FALSE WeighingFunc=GetVolume Min Radius Pixel=5 Print To } \\
\text { File=FALSE Exclude Border Objects=TRUE ToCalculate=ContactRatio CalcType = Mean }\end{array}$ \\
\hline
\end{tabular}

Parameters I to 6 are kinetic; 7 to 23 are static.

quality. The concentration of Hoechst was optimized to obtain reliable segmentation without inducing cell death, and cells were imaged with a 380/10-nm pass band filter with a $50 \%$ neutral density filter to reduce UV exposure (see Materials and Methods). To obtain a sufficient number of cells per condition for analysis, we acquired four movies per well.

Once the minimal acquisition parameters are determined, it is important to verify that cell viability is unaffected under those conditions. We compared the growth and survival of stained and imaged cells with unstained and nonimaged cells and showed that cell numbers were unaffected (data not shown). The light-induced damage induced by our acquisition conditions is therefore tolerable to cells and can be used for screening. In summary, for our screen, we determined that we could acquire four movies per well with 80 images at an excitation wavelength of $488 / 10 \mathrm{~nm}$, an exposure time of $150 \mathrm{~ms}$, and a frame rate of 4.5 images per second and 1 image at an excitation wavelength of $380 / 10 \mathrm{~nm}$, a $50 \%$ neutral density filter, and an exposure time of $50 \mathrm{~ms}$.

\section{Assay Validation}

Endosomes are moved by molecular motors along the tubulin and actin cytoskeleton. The tubulin network is essential for fast and directed movement and can be dissociated by microtubule depolymerizing agents such as nocodazole. ${ }^{16,17}$

To validate our acquisition parameters and experimental setup, U-2 OS cells were treated with increasing concentrations of nocodazole ranging from 0.4 to $10 \mu \mathrm{M}$ and the kinetic 


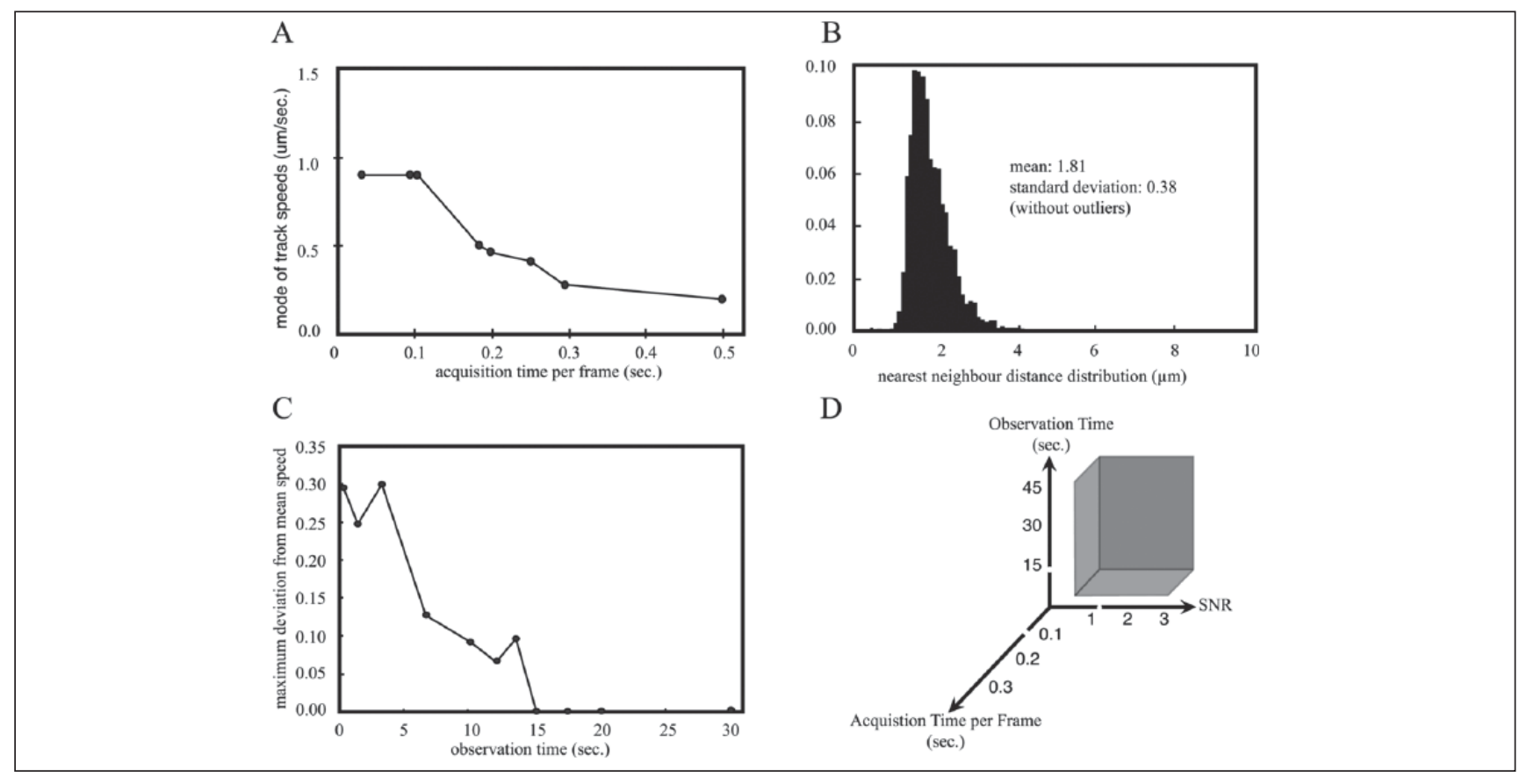

Figure I. Optimization of parameters for kinetic acquisition of endosome movement. (A) The mode vesicle speed is plotted as a function of the acquisition time per frame. The curve shows a plateau on the left-hand side indicating that from an acquisition time of $0.1 \mathrm{~s}$ (frame rate of 10 frames per second), most vesicles can be tracked successfully. (B) Nearest neighbor distance distribution, measured between the endosomes. (C) The maximum difference of the average vesicle speed measured for several movies to the mean measurement is plotted as a function of observation time. Each point is calculated on a set of four movies. The maximum deviation is used to reflect variability between similar measurements. When decreasing, it indicates reproducibility. The curve reveals the minimum movie duration to consistently measure the endosome speed. (D) Acquisition space: the gray cube delimits the acquisition space (possible values of parameter and their combination) for the critical parameter observation time, frame rate, and image signal-to-noise (SNR) ratio.

parameters of vesicles were measured (see Fig. 2A). Average endosome speed was reduced with increasing concentrations of nocodazole, reaching a minimum at $3.5 \mu \mathrm{M}$. We calculated the $\mathrm{IC}_{50}$ values of 23 parameters and the Mahalanobis distance ${ }^{18}$ of all parameters between DMSO and nocodazole. Most parameters had $\mathrm{IC}_{50}$ values below $5 \mu \mathrm{M}$; the $\mathrm{IC}_{50}$ of the Mahalanobis distance was $5 \mu \mathrm{M}$ (see Fig. 2B). We decided to use $5 \mu \mathrm{M}$ nocodazole as a control during the screen. Example videos of untreated cells, DMSO-treated cells, and nocodazole-treated cells with the tracking of the objects can be seen in Supplementary Videos S1, S2, and S3, respectively.

\section{Primary Screen of MSD Chemical Library}

We screened the MSD Spectrum Collection library comprising 2000 compounds arrayed in seven 384-well plates using our optimized acquisition parameters. The acquisition of a well requires $1 \mathrm{~min}, 30 \mathrm{~s}$; a column of 16 wells requires $24 \mathrm{~min}$; an entire 384-well plate requires 9 h, $30 \mathrm{~min}$; and the acquisition of all seven plates requires over $66 \mathrm{~h}$.

Thus, compounds cannot be added simultaneously to all plates as the last well of the last plate would be incubated for $66 \mathrm{~h}$ longer than the first well of the first plate. The screen therefore needed to be carried out one plate at a time.
A similar incubation problem arises within a plate since the acquisition of an entire plate requires $9.5 \mathrm{~h}$. To solve this problem, we added the compounds column-wise every 24 min, which corresponded to the time required for the microscope to acquire a column. By acquiring the images column-wise, the incubation difference between the first and last wells was $24 \mathrm{~min}$, and the compound addition required $9.5 \mathrm{~h}$. The plate was imaged $4 \mathrm{~h}$ following the last compound addition. In this way, each well was incubated for approximately $14 \mathrm{~h}$, and each plate was screened on a separate day. A further DMSO-only plate was screened to increase the robustness of the statistics. The entire screen took approximately a month to perform and generated 12,288 movies. A schematic workflow of the screen can be seen in Supplementary Figure S2. The images were analyzed, outliers removed, and the data normalized plate-wise to the DMSO control (see Materials and Methods).

Multivariate $\mathrm{Z}$ factors ${ }^{19}$ for six plates were between 0.77 and 0.87 , indicating strong phenotypic responses. One plate showed low multivariate $\mathrm{Z}$ factor and was discarded from further analysis. The data were filtered for outliers, toxicity, and autofluorescent compounds (see Materials and Methods). For 247 compounds, tracking failed due to either focus problems or clumping of the objects, and the data 


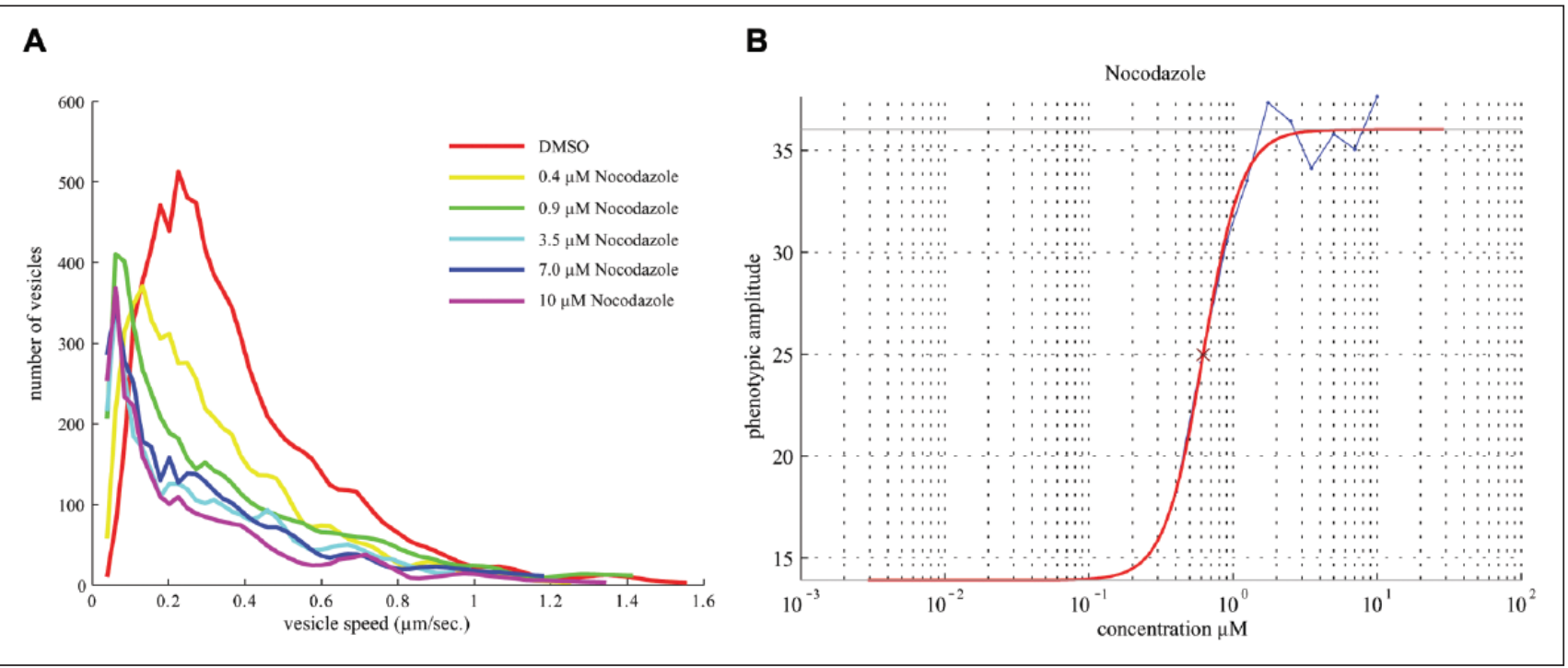

Figure 2. Assay validation on nocodazole-treated cells. (A) Probability density plot of endosome speed, with endosome speed on the $x$-axis and number of endosomes on the $y$-axis. Each color represents a different nocodazole concentration. The peak of the speed distributions is maximally left shifted at $0.9 \mu \mathrm{M}$. (B) $I C_{50}$ curve of the Mahalanobis distance between DMSO and the various concentrations of nocodazole. The Mahalanobis distance is calculated from all parameters, including parameters not influenced by nocodazole such as nuclear Hoechst intensity. The blue line connects Mahalanobis distances for each concentration; the red line is a four-parameter logistic regression fit curve for $\mathrm{IC}_{50}$.

were not further analyzed. In total, 1452 compounds could be successfully analyzed. The pairwise Pearson correlation coefficient between the multiparametric profiles of the nocodazole controls on different plates was on average 0.8 , indicating that the assay was highly reproducible. The cell counts were not significantly different between positive and negative controls, indicating that nocodazole had not caused cells to detach.

Compounds were classified as active based on their Mahalanobis distance from DMSO. ${ }^{18-21}$ We chose a Mahalanobis distance of 13 as a cutoff since it was the halfway distance between the highest untreated control (11.13) and the lowest of the nocodazole control (14.9) (Fig. 3A). Our selected cutoff was very stringent as neither DMSO or untreated false positives nor nocodazole false negatives were selected. This cutoff selected 79 compounds out of 1452 reagents (5.4\%). The normalized well-averaged data are shown in Supplementary Table S2.

Next, we performed hit confirmation with 73 of 79 hits in duplicate. Not all primary hits were tested in the reconfirmation, because a previous analysis (data not shown) had identified a slightly different subset of compounds, and hit picking had been carried out based on a common list. Fortyeight compounds showed in both duplicates a Mahalanobis distance greater than the highest Mahalanobis distance of the untreated control, resulting in a $65.7 \%$ reconfirmation rate (Fig. 3B). Forty-eight compounds showed a Pearson correlation coefficient above 0.6 between replicate runs and were therefore considered robust and reconfirmed (reconfirmation rate of 65.7\%). The normalized well-averaged data are shown in Supplementary Table S3.

\section{Clustering of Confirmed Hits}

Next, we analyzed the phenotypes of our hit compounds. All compounds showed both a static and a kinetic phenotype, and none of the identified compounds would have been missed in a nonkinetic screen. This was to be expected since changes in exchange rates between donor and acceptor compartments would most likely lead to morphological changes in those compartments. We performed hierarchical clustering of the hit phenotypes using the cosine as a distance measure. To estimate the importance of the kinetic data for distinguishing phenotypes, clustering was carried out with (Suppl. Fig. S2) and without (Suppl. Fig. S3) kinetic parameters. Most compounds resulted in decreased endosome motility, but a small cluster showed increased speed and displacement of endosomes (see cluster delimited by green box in Suppl. Fig. S2). This cluster contains oleandrin, digitoxin, digoxin, and neriifolin, all of which belong to the class of cardiac glycosides. These compounds do not cluster together clearly without kinetic parameters. We performed also clustering around medoids to confirm this result. Silhouette analysis of the data set without the kinetic parameter suggested an optimum of 17 clusters. ${ }^{22}$ With 17 clusters, the four cardiac glycosides did not cluster together, but with the kinetic parameter, they formed a distinct cluster (Suppl. Tables S4 and S5, respectively). Next, we performed principal component 


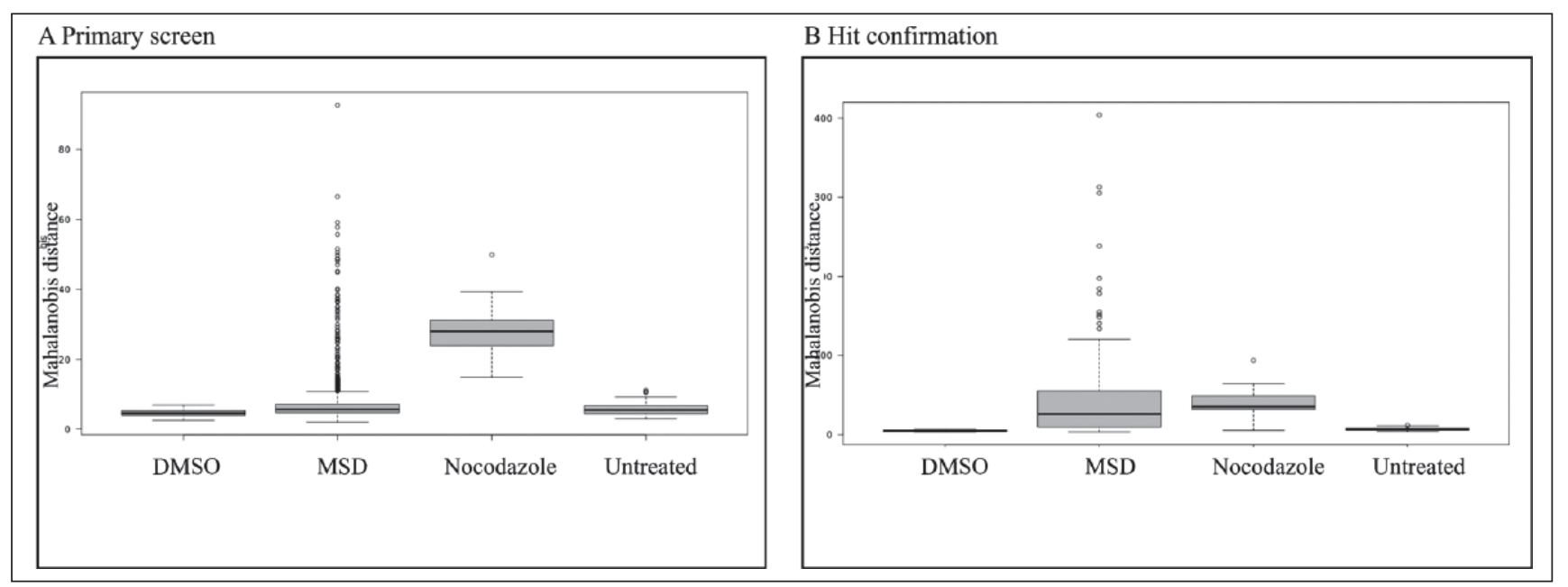

Figure 3. Boxplots with Mahalanobis distance per group of treatment. (A) Mahalanobis distance was calculated for each well from all parameters for the primary screen. (B) Mahalanobis distance was calculated for each well from all parameters for the reconfirmation screen. DMSO-treated wells were used as a reference in both cases. MSD represents library compound-treated wells.

analysis (PCA) to estimate the contribution of the kinetic parameters to the clustering results. Kinetic parameters contributed largely to principal components 1 and 2 (Fig. 4A). Kinetic parameter 3 (maximum displacement) showed the strongest contribution to principal component 2 , with a Pearson correlation between principal component 2 and kinetic parameter 3 of -0.8 (Fig. 4B). Thus, kinetic parameters have an important contribution to the clustering result.

As the results of hierarchical clustering can depend on multiple parameter choices, we also performed an additional, independent test not relying on the clustering of the phenotypes. Chemically similar compounds can in many cases be expected to induce similar phenotypes and therefore show a lower phenotypic distance. We computed the Tanimoto similarities between hit compounds using 2D chemical fingerprints from the Chemistry Development Kit. ${ }^{23}$ A group of 19 chemical pairs was observed to be clearly more similar to each other than the other compound pairs with Tanimoto distances between 0.74 and 1 versus less than 0.61 for others (annotated with color blocks in Suppl. Fig. S4). We then compared pairwise phenotypic Euclidian distances between similar and dissimilar compound pairs. As predicted, on average, the distances were smaller between similar compounds than between similar and dissimilar compounds. The difference between the distances of both groups can be expected to increase for more accurate phenotypic descriptors. We tested the difference of distances between the two groups with a one-sided Kolmogorov-Smirnov test. The distance was greater and better with all parameters, including the kinetic parameters $(p=$ $4 \mathrm{e}-5)$ compared with the static parameters only $(p=2.5 \mathrm{e}-4)$ (see Suppl. Fig. S4). In summary, the kinetic parameters allow a more precise phenotypic clustering of distinguishing compounds, causing an increase in kinetic parameters compared with compounds reducing kinetic parameters. The clustering with kinetic parameters was more consistent with the clustering of the chemical structures.

\section{Discussion}

Our aim was to develop a high-content assay measuring the kinetic parameters of endosomal movement, with emphasis on going beyond the more commonly measured static parameters, such as morphology and intensity. The efforts to develop, carry out, and analyze the screen were considerable and in our experience much more difficult than for a conventional screen with fixed cells. First, object tracking requires the optimization of acquisition parameters, such as frame rate and movie length, that is not required for fixed assays. This optimization requires a suite of experiments where cells are imaged at a different frame rate and for long periods and the survival of the cells under optimized conditions is verified. Second, the throughput of the assay is massively reduced due to the time required to add compounds in a synchronized fashion and to acquire a plate. With our equipment and protocol, the acquisition of a single 384-well plate requires $10 \mathrm{~h}$ of recording. This imposes a compound addition time of $10 \mathrm{~h}$, and therefore a single plate per day can maximally be acquired. The throughput could be increased with additional microscopes or a much faster microscope with a larger camera chip but would still be much lower than a conventional fixed-cell screen. Third, incubation conditions are not ideal in a microscope. This is particularly true for the BD Pathway 855, which does not have humidity control. We circumvented evaporation problems by covering the wells with mineral oil and using $\mathrm{CO}_{2}$ independent medium to compensate the diminished gas exchange. Fourth, the image analysis to segment all frames of the movies and link the objects between frames is challenging and computationally demanding. The computational costs are dependent on the software, the desired precision of the analysis, and the hardware 

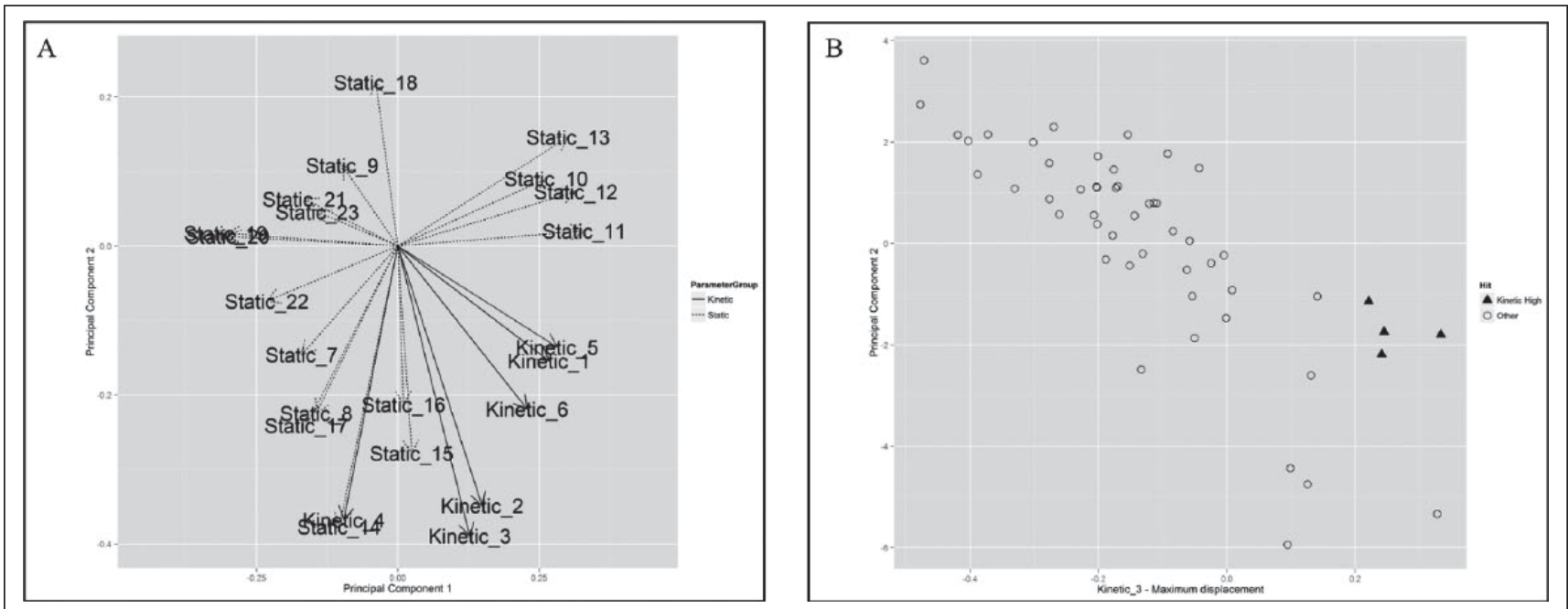

Figure 4. Principal component analysis. (A) Plot showing loading of each parameter onto principal component I (PCI) on the $x$-axis and principal component 2 (PC2) on the $y$-axis. Kinetic parameters are represented with a normal line and static parameters with dotted line. This indicates that kinetic parameters I, 5, and 6 greatly contribute to $\mathrm{PCl}$, and kinetic parameters 2,3 , and 4 contribute greatly to PC2. (B) Scatter plot of kinetic parameter 3 and PC2 showing high anticorrelation. Pearson correlation is -0.8 . Cardiac glycoside compounds are marked as black triangles.

available at the time of analysis. Our software, Motion Tracking, segments and tracks objects with high precision and requires correspondingly large computational resources. The processing time of a single 384-well plate required 43,000 CPU hours and was carried out on a $2500 \mathrm{CPU}$ cluster farm of the Technische University of Dresden.

Thus, live-cell screens tracking numerous and fast objects are challenging, and the benefits of obtaining kinetic parameters must be carefully evaluated in light of the increased effort and costs involved.

Our analysis shows that the kinetic parameters did not contribute to the detection of hit phenotypes. Therefore, in our case, a large chemical screen could and should be carried out using fixed cells. Furthermore, our analysis showed that kinetic parameters contribute critical additional information for clustering of hit compounds. The kinetic parameters allow two classes of compounds to be distinguished: those that decrease and those that increase the motility of endosomes. The static morphological changes do not result in a clear distinction between these two classes. A further indication that the use of kinetic parameters is beneficial for phenotypic clustering is that the phenotypic clustering results are more consistent with the chemical clustering when including the kinetic parameters. Thus, even though our kinetic assay would not be amenable to a large compound screen, it is of great value for classifying compounds that would emerge from such a screen. In general, increasing the number of relevant and orthogonal parameters to measure cellular phenotypes allows more granular resolution of phenotypes and therefore better clustering.

Our kinetic analysis allowed us to distinguish a class of compounds that increased the kinetic parameters such a speed and processivity. All compounds belong to the family of cardiac glycosides, whose primary mode of action is the inhibition of $\mathrm{Na}^{+} / \mathrm{K}^{+}$ATPases. Rosen et al. ${ }^{24}$ have previously shown that cardiac glycosides had a profound effect on endocytosis and that transferrin accumulated in large perinuclear vesicles positive for Rab7 and Rab11 but negative for low-density lipoprotein. This effect was mediated directly by $\mathrm{Na}^{+} / \mathrm{K}^{+}$ATPase activity. It was proposed that the cardiac glycosides alter the activity of $\mathrm{Na}^{+} / \mathrm{K}^{+}$pump in endosomes, thereby increasing the acidification of endosomes. ${ }^{25}$ The phenotype induced by the cardiac glycosides in U-2 OS cells appears to be different from the one reported by Rosen and coworkers. In some cells, large perinuclear vesicles can be observed, but the majority of cells have small, fast-moving Lamp1-GFP vesicles. The differences might be due to differences in cell lines, different concentration of drugs, or incubation time. At this stage, we cannot speculate as to the reasons for observed increased movement of Lamp1 endosomes in response to cardiac glycosides. Cardiac glycosides have been reported to have pleiotropic effects, affecting many cellular pathways ${ }^{26}$; in particular, nonreceptor tyrosine kinase $\mathrm{c}-\mathrm{Src}$ has been reported to be activated in response to binding to $\mathrm{Na}^{+} / \mathrm{K}^{+}$ pumps. Src family kinases have been implicated in regulating endosomal trafficking through control of distinct Rho family GTPases and the actin cytoskeleton. ${ }^{27}$ Src associates with early endosomes containing RhoD and with late endosomes containing RhoB. ${ }^{28,29}$ Thus, it is possible that the increased motility of late endosomes upon treatment with cardiac glycoside is a result of the activation of c-Src and the activation of RhoD containing late endosomes. 
Here we bring clear evidence that a kinetic screen improves phenotypic clustering of hit compounds and brings more insight into their modes of action. Due to the low throughput, this kind of assay is not suitable for primary screens but can be added to the secondary assay pipelines and hit confirmations.

\section{Acknowledgments}

We are grateful to Ina Nüsslein (MPI-CBG) for fluorescenceactivated cell sorting of Lamp-1-GFP cells and to Esteban C. Dell'Angelica (UCLA) for providing Lamp1-GFP plasmid.

\section{Declaration of Conflicting Interests}

The authors declared no potential conflicts of interest with respect to the research, authorship, and/or publication of this article.

\section{Funding}

The authors disclosed receipt of the following financial support for the research, authorship, and/or publication of this article: The research was funded by the German Ministry of Education and Research (BMBF) within the Framework Concept "Quantpro" (0313831A) and managed by the Project Management Agency Jülich.

\section{References}

1. Antczak, C.; Takagi, T.; Ramirez, C. N.; et al. Live-Cell Imaging of Caspase Activation for High-Content Screening. J. Biomol. Screen. 2009, 14, 956-969.

2. Cervantes, S.; Prudhomme, J.; Carter, D.; et al. High-Content Live Cell Imaging with RNA Probes: Advancements in HighThroughput Antimalarial Drug Discovery. BMC Cell Biol. 2009, 10, 45.

3. Neumann, B.; Held, M.; Liebel, U.; et al. High-Throughput RNAi Screening by Time-Lapse Imaging of Live Human Cells. Nat. Methods 2006, 3, 385-390.

4. Schulte, J.; Sepp, K. J.; Wu, C.; et al. High-Content Chemical and RNAi Screens for Suppressors of Neurotoxicity in a Huntington's Disease Model. PloS One 2011, 6, e23841.

5. Chen, J. W.; Murphy, T. L.; Willingham, M. C.; et al. Identification of Two Lysosomal Membrane Glycoproteins. J. Cell Biol. 1985, 101, 85-95.

6. Lewis, V.; Green, S. A.; Marsh, M.; et al. Glycoproteins of the Lysosomal Membrane. J. Cell Biol. 1985, 100, 1839-1847.

7. Platta, H. W.; Stenmark, H. Endocytosis and Signaling. Curr. Opin. Cell Biol. 2011, 23, 393-403.

8. Collinet, C.; Stoter, M.; Bradshaw, C. R.; et al. Systems Survey of Endocytosis by Multiparametric Image Analysis. Nature 2010, 464, 243-249.

9. Frosio, I.; Lucchese, M.; Borghese, N. A. A New and Reliable Poisson Noise Estimator for Radiographic Images; 14th International Conference on Image Analysis and Processing, Proceedings. New York, NY: 2007; pp 725-730.

10. Rink, J.; Ghigo, E.; Kalaidzidis, Y.; et al. Rab Conversion as a Mechanism of Progression from Early to Late Endosomes. Cell 2005, 122, 735-749.
11. Marsico, G. Analysis of the Interplay between Endocytosis and Cell Morphology through the Development of Computational Methods. Ph.D. Thesis, Technische Universität Dresden, 2011.

12. Kalaidzidis, Y. Intracellular Objects Tracking. Eur. J. Cell Biol. 2007, 86, 569-578.

13. Birmingham, A.; Selfors, L. M.; Forster, T.; et al. Statistical Methods for Analysis of High-Throughput RNA Interference Screens. Nat. Methods 2009, 6, 569-575.

14. Nadon, R.; Malo, N.; Hanley, J. A.; et al. Statistical Practice in High-Throughput Screening Data Analysis. Nat. Biotechnol. 2006, 24, 167-175.

15. Falcon-Perez, J. M.; Nazarian, R.; Sabatti, C.; et al. Distribution and Dynamics of Lamp1-Containing Endocytic Organelles in Fibroblasts Deficient in BLOC-3. J. Cell Sci. 2005, 118, 5243-5255.

16. Matteoni, R.; Kreis, T. E. Translocation and Clustering of Endosomes and Lysosomes Depends on Microtubules. J. Cell Biol. 1987, 105, 1253-1265.

17. Cordonnier, M. N.; Dauzonne, D.; Louvard, D.; et al. Actin Filaments and Myosin I Alpha Cooperate with Microtubules for the Movement of Lysosomes. Mol. Biol. Cell 2001, 12, 4013-4029.

18. Mahalanobis, P. C. On the Generalized Distance in Statistics. Proc. Natl. Inst. Sci. India 1936, 2, 49-55.

19. Kümmel, A.; Gubler, H.; Gehin, P.; et al. Integration of Multiple Readouts into the $Z^{\prime}$ Factor for Assay Quality Assessment. J. Biomol. Screen. 2010, 15, 95-101.

20. Ljosa, V.; Caie, P. D.; ter Horst, R.; et al. Comparison of Methods for Image-Based Profiling of Cellular Morphological Responses to Small-Molecule Treatment. J. Biomol. Screen. 2013, 18, 1321-1329.

21. Caie, P. D.; Walls, R. E.; Ingleston-Orme, A.; et al. HighContent Phenotypic Profiling of Drug Response Signatures across Distinct Cancer Cells. Mol. Cancer Ther. 2010, 9, 1913-1926.

22. Rousseeuw, P. J. Silhouettes-a Graphical Aid to the Interpretation and Validation of Cluster-Analysis. J. Comp. Appl. Math. 1987, 20, 53-65.

23. Steinbeck, C.; Han, Y.; Kuhn, S.; et al. The Chemistry Development Kit (CDK): An Open-Source Java Library for Chemo- and Bioinformatics. J. Chem. Inf. Comput. Sci. 2003, 43, 493-500.

24. Rosen, H.; Glukhman, V.; Feldmann, T.; et al. Cardiac Steroids Induce Changes in Recycling of the Plasma Membrane in Human NT2 Cells. Mol. Biol. Cell 2004, 15, 1044-1054.

25. Tomer, F.; Vladimir, G.; Eleonora, M.; et al. Role of Endosomal $\mathrm{Na}+-\mathrm{K}+-\mathrm{ATPase}$ and Cardiac Steroids in the Regulation of Endocytosis. Am. J. Physiol. Cell Physiol. 2007, 293, C885-C896.

26. Riganti, C.; Campia, I.; Kopecka, J.; et al. Pleiotropic Effects of Cardioactive Glycosides. Curr. Med. Chem. 2011, 18, 872-885.

27. Sandilands, E.; Frame, M. C. Endosomal Trafficking of Src Tyrosine Kinase. Trends Cell Biol. 2008, 18, 322-329.

28. Gasman, S.; Kalaidzidis, Y.; Zerial, M. RhoD Regulates Endosome Dynamics through Diaphanous-Related Formin and Src Tyrosine Kinase. Nat. Cell Biol. 2003, 5, 195-204.

29. Sandilands, E.; Cans, C.; Fincham, V. J.; et al. RhoB and Actin Polymerization Coordinate Src Activation with EndosomeMediated Delivery to the Membrane. Dev. Cell 2004, 7, 855-869. 